\title{
A contribution to the study of the ant fauna (Hymenoptera: Formicidae) of Eastern Iran
}

\author{
With 59 figures
}

Hassan Ghahari ${ }^{1}$, Mostafa R. Sharaf ${ }^{2}$, Abdulrahman S. Aldawood ${ }^{2}$ and Cedric Alex Collingwood ${ }^{3}$

${ }^{1}$ Department of Plant Protection, Yadegar - e- Imam Khomeini (RAH) Branch, Islamic Azad University, Tehran, Iran. hghahari@yahoo.com

${ }^{2}$ Economic Entomology Research Unit (EERU), Plant Protection Department, College of Food and Agriculture Science, King Saud University, Riyadh 11451, P. O. Box 2460 - antsharaf@gmail.com

${ }^{3} 18$ Milton Street, Skipton, North Yorkshire, BD 23 2E8, UK. - cedcollingwoodants@yahoo.co.uk

Published on 2015-12-21

\section{Summary}

A faunistic inventory of several regions of eastern Iran revealed 30 ant species belonging to 17 genera (Aphaenogaster Mayr 1853, Camponotus MaYr 1861, Cataglyphis Foerster 1850, Crematogaster Lund 1831, Dolichoderus Lund 1831, Formica Linnaeus 1758, Lasius FABricius 1804, Lepisiota SANTSCHi 1926, Linepithema MAYr 1866, Messor Forel 1890, Monomorium Mayr 1855, Plagiolepis Mayr 1861, Pachycondyla Smith 1858, Pheidole Westwood 1839, Polyrhachis Smith 1857, Solenopsis Westwood 1840, Tapinoma Foerster 1850. Two species, Crematogaster laestrygon EMERY, 1869 and Camponotus adenensis EMERY, 1893 are recorded for the first time from Iran. The majority of species were widespread taxa. No apparently endemic species were collected. Information on local and world distribution of collected species is presented.

\section{Key words}

Palearctic region, Middle East, distribution, list, new record, Iran

\section{Zusammenfassung}

Das faunistische Verzeichnis mehrerer Regionen in Ost-Iran nennt 30 Ameisenarten, die zu 17 Gattungen gehören: Aphaenogaster MaYr 1853, Camponotus MaYr 1861, Cataglyphis Foerster 1850, Crematogaster Lund 1831, Dolichoderus Lund 1831, Formica Linnaeus 1758, Lasius Fabricius 1804, Lepisiota SANTschi 1926, Linepithema MaYr 1866, Messor Forel 1890, Monomorium Mayr 1855, Plagiolepis Mayr 1861, Pachycondyla Smith 1858, Pheidole WeStwood 1839, Polyrhachis Smith 1857, Solenopsis Westwood 1840, Tapinoma Foerster 1850. Zwei Arten, Crematogaster laestrygon EMERY, 1869 und Camponotus adenensis EMERY, 1893 werden erstmals für Iran nachgewiesen. Die Mehrzahl der behandelten Taxa ist weit verbreitet. Offensichtlich endemische Arten wurden nicht gesammelt. Weiterhin wird die lokale und weltweite Verbreitung der gesammelten Arten dargestellt. 


\section{INTRODUCTION}

Iran encompasses a large portion of the Iranian Plateau (including Afghanistan and adjacent West Pakistan) and covers $1623,779 \mathrm{~km}^{2}$. It is bordered in the north by the Caucasus Mountains, Middle Asian regions, and the Caspian Sea (27 $\mathrm{m}$ below sea level); in the west by the Anatolian and Mesopotamian regions; in the east by the eastern portion of the Iranian Plateau and the BaluchSindian Region, and finally in the south by the Persian Gulf and Oman Sea, which are connected by the latter to the Indian Ocean (ZeHzad et al. 2002).

Among the wide variety of animal biodiversity on earth, ants or the Formicidae are a well-recognized cosmopolitan group of insects (HöLldobler \& WiLsON 1990). Ants include about $1.5 \%$ of the total known world entomofauna, but comprises more than $10 \%$ of the animal biomass of grasslands, tropical forests, and other major habitats (Wilson 2000). The nesting habits of ants are extremely diverse, including soil, leaf litter, rotting logs, and in various plants parts (e.g. roots, stems fruits) (HöLldobler \& Wilson 1990). There are about 15,097 valid species and subspecies belonging to 16 valid extant subfamilies and 402 recognized genera (http://www.antwiki.org/).

The ant fauna of Iran has not been well studied especially considering the vast area of the country and the countless uncollected areas. Several papers on the Iranian Formicidae have been published reporting at least 144 species and subspecies (Ardeh 1994; Tirgari \& Paknia 2004; Paknia \& Tirgari 2004; PAKnia 2006; PAKNia \& Kami 2007; PAKnia et al. 2008, 2010; RAfINEJAD et al. 2009; RAdChEnKo \& PAKNia 2010; GHAhari \& CollingWOOD 2011, 2013; GHAHARI et al. 2008, 2009, 2010a, b, 2011; Firouzi et al. 2011; Mohammadi et al. 2012; SHIRAN et al. 2013). The objective of this work is to report for the first time on ants the eastern region of Iran.

\section{Materials and Methods}

Study area: In this study, ants were collected in eastern Iran including the following Provinces, North Khorasan, South Khorasan, Kerman, Razavi Khorasan, Sistan, and

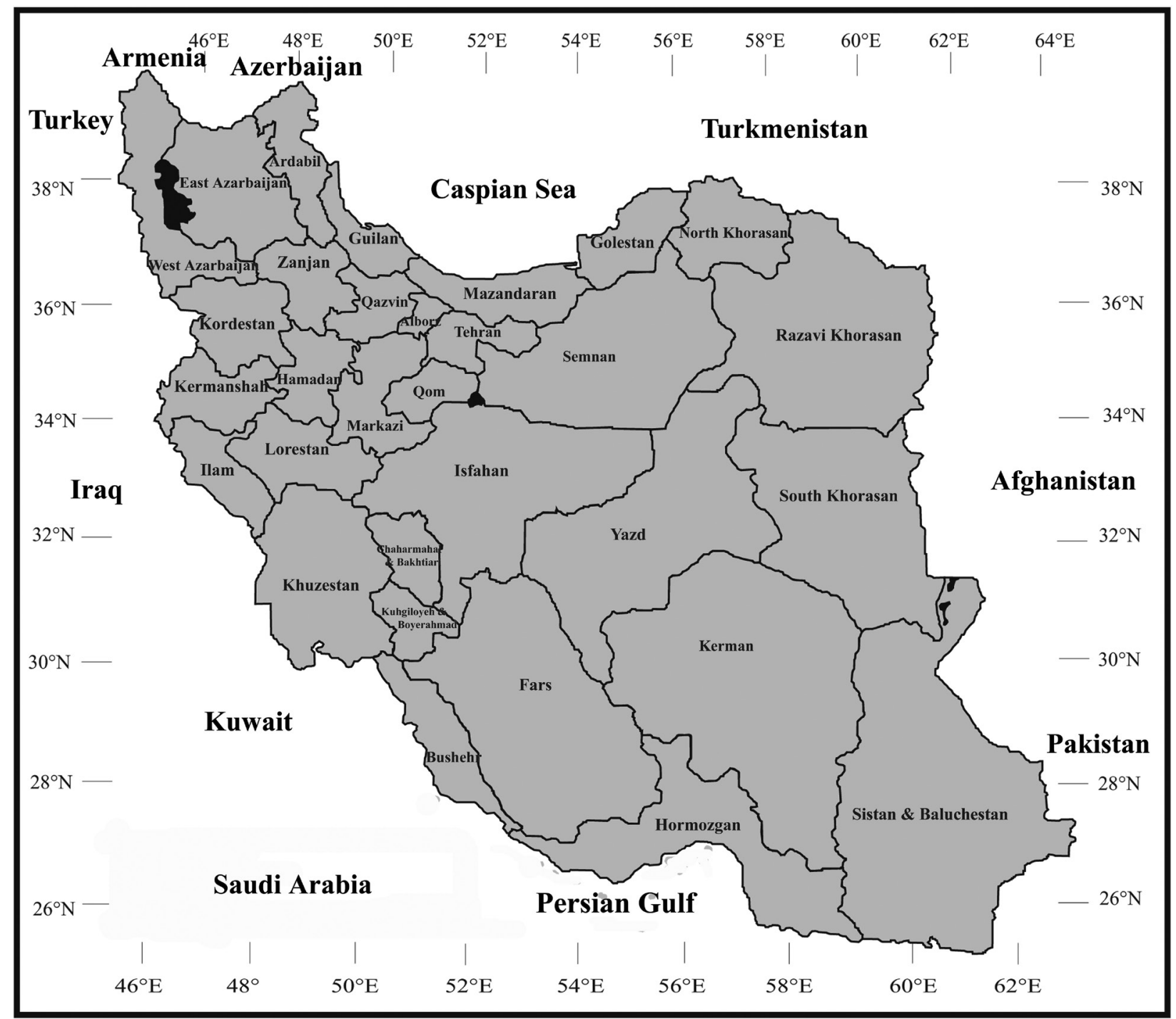

Fig. 1: Map of Iran with boundaries of Provinces for showing the distribution of ant species of this paper. 
Baluchestan (Fig. 1). Khorasan Province is in northeastern Iran, but historically has been referred to a much larger region east and northeast of the original Persian Empire. Khorasan was the largest Province of Iran, but was divided in 2004 into three provinces, North Khorasan $\left(37.4761^{\circ} \mathrm{N}\right.$ $\left.57.3317^{\circ} \mathrm{E}\right)$, South Khorasan $\left(32.8653^{\circ} \mathrm{N} 59.2164^{\circ} \mathrm{E}\right)$, and Razavi Khorasan $\left(36.2980^{\circ} \mathrm{N} 59.6057^{\circ} \mathrm{E}\right)$. Kerman Province $\left(30.2907^{\circ} \mathrm{N} 57.0679^{\circ} \mathrm{E}\right)$, is located in southeastern Iran and is the second largest province of Iran with an area of $180,726 \mathrm{~km}^{2}$, encompassing nearly $11 \%$ of the land area of Iran. This region is largely steppe or sandy desert, although there are some oases where dates, oranges, and pistachios are cultivated. The province of Sistan and Baluchestan $\left(29.4924^{\circ} \mathrm{N} 60.8669^{\circ} \mathrm{E}\right)$ is located in the Southeast, bordering Pakistan and Afghanistan, and is the largest in Iran, with an area of $181,785 \mathrm{~km}^{2}$. All ant specimens were collected either by using pitfall traps, sweeping or hand collecting.

Illustrations: Specimens were photographed at California Academy of Science, San Francisco, USA, using a JVC KYF70B3CCD digital camera attached to a Leica M420 stereomicroscope. All digital images were processed using Auto-Montage (Syncroscopy, Division of Synoptics Ltd, USA) software. Images of the specimens are available in full color on www.antweb.org. Due to their importance in identification, images of the type specimens are presented as respective taxa.

\section{RESULTS}

\section{Subfamily Dolichoderinae}

Genus Dolichoderus Lund, 1831

\section{Dolichoderus quadripunctatus (LINNAEUS, 1771)}

(Figs 2-3)

Material examined: Razavi Khorasan Province, Neyshabour, $36^{\circ} 12^{\prime} \mathrm{N} 58^{\circ} 45^{\prime} \mathrm{E}$, (3), June-August 2010 . Handcollecting.

Iranian records: East Azarbaijan (GHAHARI et al. 2011), Guilan (PAKNIA et al. 2010).

Distribution: This species is widely distributed in numerous countries of Palearctic and West Asian regions (SHATTUCK 1994).

\section{Genus Linepithema MAYr, 1866}

Linepithema humile MAYR, 1868

(Figs 4-5)

Material examined: South Khorasan Province, Birjand, $32^{\circ} 32^{\prime} \mathrm{N} 58^{\circ} 50^{\prime} \mathrm{E},(1)$, September 2009. Handcollecting. Iranian records: Mazandaran (Ghahari et al. 2009).
Distribution: Linepithema humile is a successful invasive species, originally described from Argentina. It has been reported from many regions worldwide including the Arabian Peninsula (Collingwood et al. 1997; CollingWOOD \& VAN HARTEN 2001; Collingwood et al. 2011), Europe and the Mediterranean region (Collingwood 1979; Carpintero et al. 2005), Africa (Holway et al. 2002), and USA (WARD 1987; SuAREZ et al. 1998).

\section{Genus Tapinoma Foerster, 1850}

\section{Tapinoma cf. simrothi KRAUSSE, 1911}

(Figs 6-7)

Material examined: South Khorasan Province, Birjand, $32^{\circ} 32^{\prime} \mathrm{N} 58^{\circ} 50^{\prime} \mathrm{E},(2)$, September 2009. Sweeping. Razavi Khorasan Province, Ferdows, $34^{\circ} 10^{\prime} \mathrm{N} \quad 57^{\circ} 40^{\prime} \mathrm{E},(1)$, September-October 2012. Pitfall traps.

Iranian records: East Azarbaijan (Sakenin Chelav et al. 2008a), Isfahan (Shiran et al. 2012), Khuzestan (Dezhakam \& Soleyman Nejadiyan 2000; Mossadegh et al. 2008; Alizadeh et al. 2012; Mossadegh 2012).

Distribution: This species is distributed in Europe (SHATTUCK 1994).

\section{Subfamily Formicinae}

\section{Genus Camponotus MaYR, 1861}

\section{Camponotus adenensis EMERY, 1893 (Figs 8-9)}

Material examined: Razavi Khorasan Province, Sabzevar, $36^{\circ} 12^{\prime} \mathrm{N} 57^{\circ} 35^{\prime} \mathrm{E}$, (1), October 2009. New record for Iran. Handcollecting.

Distribution: This species was originally described from Yemen and recorded from the Kingdom of Saudi Arabia (Collingwood 1985; Collingwood \& Agosti 1996).

\section{Camponotus libanicus AndRe, 1881 (Figs 10-11)}

Material examined: Kerman Province, Kerman, $30^{\circ} 20^{\prime} \mathrm{N}$ $58^{\circ} 00^{\prime} \mathrm{E}$, (3), April 2010. Sweeping. Sistan \& Baluchestan Province, Zahedan, $29^{\circ} 29^{\prime} \mathrm{N} 60^{\circ} 15^{\prime} \mathrm{E}$, (2), May 2011. Handcollecting.

Iranian records: Khuzestan (Shiran et al. 2012, 2013), Tehran (Alipanah et al. 2000; Paknia et al. 2010).

Distribution: Cyprus, Greece, Turkey (Karaman \& AKtac 2013), Israel, Lebanon (Ionescu-Hirsch 2009). 
Comments: Associated with Aphis craccivora KocH on Rumex acetosa L. (SHiran et al. 2013).

\section{Camponotus xerxes ForeL, 1904 \\ (Figs 12-13)}

Material examined: Kerman Province, Bam, $29^{\circ} 04^{\prime} \mathrm{N}$ 58²1'E, (2), April 2010. Handcollecting. Sistan \& Baluchestan Province, Zahedan, $29^{\circ} 29^{\prime} \mathrm{N} 60^{\circ} 15^{\prime} \mathrm{E},(1)$, May 2011. Pitfall traps.

Iranian records: East Azerbaijan (Samin et al. 2011), Kermanshah, Kordestan (Ghahari \& Collingwood 2013), Khuzestan (Dezhakam \& Soleyman Nejadiyan 2000; Alizadeh et al. 2012; Mossadegh 2012), Mazandaran (Sakenin Chelav et al. 2008b; Ghahari et al. 2008, 2009, 2010b), northern Iran (Forel 1904; Ardeh 1994; Radchenko 1996, 1997).

Distribution: Arabian Peninsula (Collingwood 1985; Collingwood \& Agosti 1996; Collingwood et al. 2011), Sinai Peninsula (Sharaf 2006), Central Asia (RADCHENKo 1997), Israel (IONESCU-Hirsch 2009) and Turkey (Karaman \& AKTAC 2013).

\section{Genus Cataglyphis FöRSTER, 1850 \\ Cataglyphis cinnamomea (KaravalEV, 1910) (Figs 14-15)}

Material examined: South Khorasan Province, Birjand, $32^{\circ} 32^{\prime} \mathrm{N} 58^{\circ} 50^{\prime} \mathrm{E}$, (1), September 2009. Sweeping. Iranian records: Hormozgan (Ghahari et al. 2010a; Ghahari \& Collingwood 2011), Ilam (Ghahari \& Collingwood 2013), Khuzestan (Shiran et al. 2012, 2013), Yazd (Paknia et al. 2010).

Distribution: Middle Asia, South Kazakhestan and Afghanistan (PISARSKI 1967; RADCHENKo 1998), the Arabian Peninsula (Saudi Arabia, UAE) and North Africa (Collingwood et al. 2011).

Comments: Associated with Myzus persicae Sulzer on Beta maritima L. (SHIRAN et al. 2013).

\section{Cataglyphis setipes (ForeL, 1894)}

(Figs 16-17)

Material examined: Razavi Khorasan Province, Ferdows, $34^{\circ} 10^{\prime} \mathrm{N} 57^{\circ} 40^{\prime} \mathrm{E}$, (2), September-October 2012. Pitfall traps.

Iranian records: Khuzestan (Dezhakam \& Soleyman Nejadiyan 2000; Shiran et al. 2012, 2013), Tehran (Alipanah et al. 2000).
Distribution: This species was described from Greece and has an Asian distribution range from Caucasia to Middle Asia (ArNol'Di \& Dlussky, 1978).

\section{Cataglyphis semitonsa SANTSCHI, 1929 \\ (Figs 18-19)}

Material examined: Kerman Province, Jiroft, $28^{\circ} 50^{\prime} \mathrm{N}$ 57³5'E, (1), April 2010. Sweeping.

Iranian records: Lorestan (Ghahari \& Collingwood 2013), Mazandaran (Ghahari et al. 2009), Semnan (Mirseyed et al. 2013).

Distribution: North Africa (SANTSCHI 1929), Saudi Arabia (Collingwood 1985).

\section{Genus Formica LINNAEUS, 1758 \\ Formica glauca RuzSKY, 1896 \\ (Figs 20-21)}

Material examined: Razavi Khorasan Province, Neyshabour, $36^{\circ} 12^{\prime} \mathrm{N} 58^{\circ} 45^{\prime} \mathrm{E}$, (4), June-August 2010. Handcollecting.

Iranian records: Kermanshah, Kordestan (Ghahari \& Collingwood 2013), Mazandaran (Ghahari et al. 2008, 2009, 2010b), Tehran (Alipanah et al. 2000).

Distribution: Formica glauca was described from Russian Federation (RUzSKY, 1896), and reported from Iran (Alipanah et al. 2000, GHAhari et al. 2008, 2009, 2010b, Ghahari \& Collingwood 2013), Afghanistan, Mongolia, and China (WHeEler 1930-1931; Dlussky 1967) and several countries of Europe including Bulgaria, Germany, Poland, Romania (Atanassov \& Dlussky 1992; Seifert \& SCHULTZ 2009).

\section{Genus Lasius FABRICIUS, 1804}

\section{Lasius turcicus SANTSCHI, 1921}

(Figs 22-23)

Material examined: Razavi Khorasan Province, Mashhad, $36^{\circ} 17^{\prime} \mathrm{N} 59^{\circ} 40^{\prime} \mathrm{E}$, (1), July 2011 . Handcollecting.

Iranian records: Mazandaran (Ghahari et al. 2009; Gholami et al. 2012; Afshari et al. 2013), Tehran (Alipanah et al. 2000).

Distribution: Iran (AlipanaH et al. 2000; GHAHARI et al. 2009; Gholami et al. 2012; Afshari et al. 2013) and Turkey (SANTSCHi 1921). 
Genus Lepisiota SANTSCHI, 1926

Lepisiota karawaiewi Kuznetsov-Ugamsky, 1929 (Figs 24-25)

Material examined: Kerman Province, Bam, $29^{\circ} 04^{\prime} \mathrm{N}$ $58^{\circ} 21^{\prime} \mathrm{E},(2)$, June 2011. Pitfall traps.

Iranian records: Mazandaran (Ghahari et al. 2008, 2009, 2010b).

Distribution: Arabian Peninsula (Kuwait) and southeastern Europe (Collingwood \& Agosti 1996).

\section{Lepisiota melas EMERY, 1915}

(Figs 26-27)

Material examined: Razavi Khorasan Province, Mashhad, $36^{\circ} 17^{\prime} \mathrm{N} 59^{\circ} 40^{\prime} \mathrm{E}$, (3), July 2011. Handcollecting. Razavi Khorasan Province, Sabzevar, $36^{\circ} 12^{\prime} \mathrm{N} 57^{\circ} 35^{\prime} \mathrm{E}$, (1), October 2009. Sweeping.

Iranian records: Fars (Mohammadi et al. 2012).

Distribution: Lepisiota melas was described from Greece (EMERY 1915) and recorded from Iran (МоHAMMADI et al. 2012) and the former Yugoslavia (Agosti \& Collingwood 1987).

\section{Genus Plagiolepis (MaYR, 1861)}

Plagiolepis cf. pygmaea (LATREILLE, 1798)

(Figs 28-29)

Material examined: Kerman Province, Kerman, $30^{\circ} 20^{\prime} \mathrm{N}$ $58^{\circ} 00^{\prime} \mathrm{E}$, (4), April 2010. Sweeping. Razavi Khorasan Province, Neyshabour, $36^{\circ} 12^{\prime} \mathrm{N} 58^{\circ} 45^{\prime} \mathrm{E}$, (1), June-August 2010. Pitfall traps.

Iranian records: Khuzestan (Ghahari et al. 2010a; Ghahari \& Collingwood 2011).

Distribution: This species was reported from southern Europe, North Africa, the Middle East, Maltese Islands (Collingwood 1985), the Arabian Peninsula (Saudi Arabia) (Collingwood \& Agosti 1996).

\section{Plagiolepis schmitzii FOREL, 1895}

(Figs 30-31)

Material examined: Sistan \& Baluchestan Province, Zahedan, (2), May 2011. Handcollecting. Kerman Province, Rafsanjan, $30^{\circ} 30^{\prime} \mathrm{N} 55^{\circ} 40^{\prime} \mathrm{E}$, (1), June 2011. Handcollecting.

Iranian records: Fars (Mohammadi et al. 2012).

Distribution: This species was described from Madeira Island (Portugal) (Forel 1895), and has been reported from North Africa (SANTSCHI 1911) and southwestern Europe and Iberian Peninsula (Collingwood 1976, 1985; Sharaf 2006), the Arabian Peninsula (Saudi Arabia) (CollingwOod 1985).

\section{Genus Polyrhachis SMITH, 1857}

Polyrhachis lacteipennis SMITH, 1858

(Figs 32-33)

Material examined: Kerman Province, Jiroft, $28^{\circ} 50^{\prime} \mathrm{N}$ $57^{\circ} 35^{\prime} \mathrm{E}$, (2), April 2010. Handcollecting. Sistan \& Baluchestan Province, Zahedan, (1), May 2011. Handcollecting.

Iranian records: Fars (Tirgari \& Paknia 2004), Khuzestan (Dezhakam \& Soleyman Nejadiyan 2000; Shiran et al. 2012, 2013), Mazandaran (Ghahari et al. 2008, 2009, 2010b), Khuzestan (Modarres Awal 1997; Mossadegh et al. 2008)

Distribution: Polyrhachis lacteipennis was originally described from India (SмIтн 1858) and reported from the Arabian Peninsula (Saudi Arabia, Oman, Yemen) and the Middle East (Collingwood \& Agosti 1996).

Comments: Associated with A. gossypii on Althaea rosae L., A. gossypii on H. Rosasinensis, and R. maidis on Bromus sp. (Shiran et al. 2013). Predator of Sesamia nonagrioides LefÉBVRe (Lepidoptera: Noctuidae) (Modarres Awal 1997).

\section{Subfamily Myrmicinae}

Genus Aphaenogaster MAYR, 1853

Aphaenogaster splendida, Rogen, 1859

(Figs 34-35)

Material examined: Razavi Khorasan Province, Mashhad, $36^{\circ} 17^{\prime} \mathrm{N} 59^{\circ} 40^{\prime} \mathrm{E}$, (1), July 2011 . Handcollecting. Iranian records: Fars (Mohammadi et al. 2012).

Distribution: Palearctic Region, southern Europe, Syria, and North Africa (Tunisia, Algeria) (EMERY 1908).

\section{Genus Crematogaster Lund, 1831 \\ Crematogaster laestrygon EMERY, 1869 (Figs 36-37)}

Material examined: Sistan \& Baluchestan Province, Iranshahr, $27^{\circ} 34^{\prime} \mathrm{N} 59^{\circ} 53^{\prime} \mathrm{E}$, (2), April 2012. New record for Iran. Handcollecting. 
Distribution: This species has a broad distribution in North Africa (Collingwood 1985, Sharaf 2006) and recorded from the Afrotropical region of the Arabian Peninsula including, Asir Mountains of the Kingdom of Saudi Arabia (Collingwood 1985) and Yemen (Collingwood \& Agosti 1996).

\section{Crematogaster luctans FoREL, 1907}

(Figs 38-39)

Material examined: Razavi Khorasan Province, Neyshabour, $36^{\circ} 12^{\prime} \mathrm{N} 58^{\circ} 45^{\prime} \mathrm{E}$, (1), June-August 2010. Handcollecting.

Iranian records: Bushehr (Ghahari et al. 2010a; Ghahari \& Collingwood 2011), Ilam (Ghahari \& Collingwood 2013).

Distribution: Iran (GHAhari et al. 2010a; GHAHARI \& Collingwood 2011, 2013), the Arabian Peninsula (Saudi Arabia) (Collingwood 1985; Collingwood \& Agosti 1996) and Africa (Forel 1907).

\section{Crematogaster subdentata MaYR, 1877 (Figs 40-41)}

Material examined: Kerman Province, Jiroft, $28^{\circ} 50^{\prime} \mathrm{N}$ 573'ㄹ, (1), April 2010. Handcollecting.

Iranian records: West Azarbaijan (Ghahari et al. 2011), Mazandaran (Ghahari et al. 2009), North of Iran (Ardeh 1994).

Distribution: Palearctic region (MAYR 1877; GHAHARI et al. 2011; Arakelian 1994).

\section{Genus Messor ForeL, 1890}

\section{Messor denticulatus SANTSCHI, 1927}

(Figs 42-43)

Material examined: Razavi Khorasan Province, Mashhad, $36^{\circ} 17^{\prime} \mathrm{N} 59^{\circ} 40^{\prime} \mathrm{E},(2)$, July 2011 . Handcollecting. Iranian records: Kordestan (Ghahari \& Collingwood 2013), Mazandaran (Ghahari et al. 2009; Gholami et al. 2012), Northeast of Iran (Arnol'di 1977).

Distribution: Kazakhstan (SANTSCHI 1927a), Turkmenistan (DLUSSKY et al. 1990).

\section{Messor meridionalis (ANDRÉ, 1883)}

(Figs 44-45)

Material examined: Kerman Province, Rafsanjan, $30^{\circ} 30^{\prime} \mathrm{N} 55^{\circ} 40^{\prime} \mathrm{E},(3)$, June 2011. Pitfall traps.
Iranian records: Sistan \& Baluchestan (Ghahari et al. 2010a; Ghahari \& Collingwood 2011), North and Southwest of Iran (Ardeh 1994).

Distribution: Palearctic Region: Central Asia, Middle East, Arabian Peninsula (Saudi Arabia, Kuwait, UAE) (Collingwood 1985; Collingwood \& Agosti 1996).

\section{Messor orientalis EMERY, 1896}

(Figs 46-47)

Material examined: Kerman Province, Kerman, $30^{\circ} 20^{\prime} \mathrm{N}$ $58^{\circ} 00^{\prime} \mathrm{E},(2)$, April 2010. Sweeping.

Iranian records: Zanjan (Hossein Nezhad et al. 2012).

Distribution: Central Asia, Middle East, South East Europe (Toнmé \& Tohmé 1981; Collingwood 1985; Collingwood \& Agosti 1996).

\section{Genus Monomorium MaYR, 1855}

\section{Monomorium areniphilum SANTSCHI, 1911 \\ (Figs 48-49)}

Material examined: South Khorasan Province, Birjand, $32^{\circ} 32^{\prime} \mathrm{N} 58^{\circ} 50^{\prime} \mathrm{E},(1)$, September 2009. Handcollecting. Iranian records: Mazandaran (Ghahari et al. 2009).

Distribution: Palearctic Region, North Africa (Collingwood 1985), Arabian Peninsula (Saudi Arabia, Kuwait, Oman, Yemen) (Collingwood \& Agosti 1996), Afrotropical region (Sudan, Mali, Niger, Senegal) (BoLton 1987).

\section{Monomorium barbatulum MAYR, 1877}

Material examined: Razavi Khorasan Province, Neyshabour, $36^{\circ} 12^{\prime} \mathrm{N} 58^{\circ} 45^{\prime} \mathrm{E}$, (2), June-August 2010. Handcollecting. Razavi Khorasan, Mashhad, $36^{\circ} 17^{\prime} \mathrm{N}$ $59^{\circ} 40^{\prime} \mathrm{E}$, (1), July 2011. Handcollecting. Sistan and Baluchestan provinces, Zahedan, (2), May 2011. Handcollecting.

Iranian records: Ardabil (Ghahari et al. 2011), South Khorasan (Tabas) (Paknia et al. 2010).

Distribution: Arabian Peninsula (United Arab Emirates) and Central Asia (Collingwood \& Agosti 1996; Collingwood et al. 2011).

\section{Monomorium salomonis (LINNAEUS, 1758) (Figs 50-51)}

Material examined: Kerman Province, Jiroft, $28^{\circ} 50^{\prime} \mathrm{N}$ 57³5'E, (4), April 2010. Handcollecting. 
Iranian records: Hormozgan (Ghahari et al. 2010a; Ghahari \& Collingwood 2011), Khuzestan (Modarres Awal 1997), Southern Iran (Menozzi 1927).

Distribution: Arabian Peninsula (Kingdom of Saudi Arabia, Kuwait) and North Africa (Collingwood 1985; Collingwood \& Agosti 1996).

Comments: Predator of Earias insulana BoISDUVAL (Lepidoptera: Noctuidae) (ModArRes AwAL 1997).

\section{Genus Pheidole Westw00D, 1839}

Pheidole megacephala FABRICIUS, 1793

(Figs 52-53)

Material examined: Kerman Province, Kerman, 30²0'N $58^{\circ} 00^{\prime}$ E, (2), April 2010. Pitfall traps. Kerman Province, Jiroft, $28^{\circ} 50^{\prime} \mathrm{N} 57^{\circ} 35^{\prime} \mathrm{E}$, (1), April 2010. Pitfall traps.

Iranian records: Hormozgan (Ghahari et al. 2010a; Ghahari \& Collingwood 2011), Kordestan (Ghahari \& Collingwood 2013), Mazandaran (Ghahari et al. 2008, 2009, 2010b).

Distribution: A cosmopolitan tramp species (Collingwood 1985; Collingwood \& Agosti 1996).

\section{Pheidole sculpturata MAYR, 1866}

(Figs 54-55)

Material examined: Razavi Khorasan Province, Mashhad, $36^{\circ} 17^{\prime} \mathrm{N} 59^{\circ} 40^{\prime} \mathrm{E}$, (3), July 2011. Sweeping. Iranian records: Fars (Mohammadi et al. 2012).

Distribution: Afrotropical region (ARNOLD 1920), Yemen (Collingwood \& Agosti 1996).

\section{Genus Solenopsis WeSTW00D, 1840}

Solenopsis wolfi EMERY, 1915

(Figs 56-57)

Material examined: Razavi Khorasan Province, Neyshabour, $36^{\circ} 12^{\prime} \mathrm{N} 58^{\circ} 45^{\prime} \mathrm{E}$, (1), June-August 2010 Sweeping.

Iranian records: Mazandaran (Ghahari et al. 2009).

Distribution: Palearctic Region, Europe (Agosti \& Collingwood 1987).

\section{Subfamily Ponerinae}

Genus Mesoponera Smith, 1858

Mesoponera ambigua (ANDRÉ, 1890)

(Figs 58-59)

Material examined: Kerman Province, Rafsanjan, $30^{\circ} 30^{\prime} \mathrm{N} 55^{\circ} 40^{\prime} \mathrm{E}$, (2), June 2011. Sweeping.

Iranian records: Khuzestan (Ghahari et al. 2010a; Ghahari \& Collingwood 2011).

Distribution: This species was described from Sierra Leon (ANDRÉ, 1890), and was recorded from the Arabian Peninsula (Saudi Arabia) (Collingwood \& Agosti 1996).

\section{Discussion}

The ant fauna of most Iranian provinces have not been studied comprehensively. Several papers have been published for ants occurring in northern Iran (in rice fields) (GHAHARI et al. 2010b), southern Iran (GHAHARI \& Collingwood 2011), northwestern Iran (Arasbaran) (GHAhARI et al. 2011), and western Iran (GHAhARI \& Collingwood 2013). Future collecting activities in the eastern region of Iran and as well in other areas will add additional taxa to the ant fauna of this country. This list of species along with the color images of each species should be a useful identification guide for this large region of Iran.

\section{Acknowledgements}

This project was supported by King Saud University, Deanship of Scientific research, College of Food and Agriculture Sciences, Research Center. The authors are grateful to Steve Shattuck (Australia) and Kadri Kiran (Turkey) for providing the necessary papers. We are grateful to Boris Kondratieff (Colorado State University) for valuable suggestions. Thanks go to Brian Fisher (California Academy of Sciences) for generous permission to use species images from Antweb. We are grateful to the M. Sc. students of the senior author, M. Toloinasab, A. Mahroughan, H. Naderian, F. Jalali (Garmsar Islamic Azad University) for cooperation in the field work.

\section{References}

Afshari, A.; Gholami, E.; Mafi Pashakolaei, Sh. A. \& Abdollahi, GH. A. 2013: Impact of Lasius turcicus (Hymenoptera: Formicidae) on efficacy of vedalia beetle, Rodalia cardinalis (Coleoptera: Coccinellidae) in citrus orchards of Mazandaran Province. - Plant Pests Research 3 (2): 53-65 [in Persian with English summary]. 
Agosti, D. \& Collingwood, C. A. 1987: A provisional list of the Balkan ants (Hym. Formicidae) and a key to the worker caste. I. Synonymic list. - Mitteilungen der Schweizerischen Entomologischen Gesellschaft 60: $51-62$.

Alipanah, H.; Kharazi-Pakdel, A. \& Moghadassi, P. 2000: Taxonomical study of Formicinae ants in Tehran. - Proceedings of 14th Iranian Plant Protection Congress, Isfahan University of Technology: p. 350.

Alizadeh, M. S.; $\quad$ Esfandiari, M.; Mossadegh, M. S.; MashayeKhi, M. \& Mohammadi, Sh. 2012: Symbiotic ants with pink hibiscus mealybug Maconellicoccus hirsutus (Green) (Hom.: Pseudococcidae) in Ahvaz. Proceedings of 20th Iranian Plant Protection Congress, University of Shiraz: p. 145.

ANDRÉ, E. 1890: Matériaux pour servir à la faune myrmécologique de Sierra-Leone (Afrique occidentale). - Revue d'Entomologie (Caen) 9: 311-327.

Arakelian, G. R. 1994: Fauna of the Republic of Armenia. Hymenopterous insects. Ants (Formicidae). - Erevan Gitutium: 153 pp.

Ardeh, M. 1994: Study on systematic and behavioral specialty of ants of Karaj region. - M. Sc thesis of Tehran University: 108 pp. (in Persian).

Arnold, G. 1920: A monograph of the Formicidae of South Africa. Part IV. Myrmicinae. - Annals of the South African Museum 14: 403-578.

ArNol'DI, K. V. 1977: Review of the harvester ants of the genus Messor (Hymenoptera, Formicidae) in the fauna of the USSR. [in Russian]. - Zoologicheskii Zhurnal 56: 1637-1648.

Arnol'di, K. V. \& Dlussky, G. M. 1978: Superfam. Formicoidea. 1. Fam. Formicidae - ants. [In Russian], pp. 519-556. - In: Medvedev, G. S. (ed.) 1978: Keys to the insects of the European part of the USSR. Vol. 3. Hymenoptera. Part 1. [In Russian]. - Opredeliteli Faune SSSR 119: 3-584.

Atanassov, N. \& Dlussky, G. M. 1992: Fauna of Bulgaria. Hymenoptera, Formicidae. - Fauna na Bûlgariya 22: 1-310.

Bolton, B. 1987: A review of the Solenopsis genusgroup and revision of Afrotropical Monomorium MaYr (Hymenoptera: Formicidae). - Bulletin of the British Museum (Natural History) Entomology 54: 263-452.

Carpintero, S.; Reyes-López, J. \& Arias de Reyna, L. 2005: Impact of Argentine ants (Linepithema humile) on an arboreal ant community in Doñana National Park, Spain. - Biodiversity and Conservation 14: 151-163.

Collingwood, C. A. 1976: A provisional list of Iberian Formicidae with a key to the worker caste (Hym. Aculeata). - EOS Revista Espanola de Entomologia, 52: 65-95.

Collingwood, C. A. 1979: The Formicidae (Hymenoptera) of Fennoscandia and Denmark. - Fauna Entomologica Scandinavica 8: 1-174.
Collingwood, C. A. 1985: Hymenoptera: Fam. Formicidae of Saudi Arabia. - Fauna of Saudi Arabia 7: 230-302.

Collingwood, C. A. \& Agosti, D. 1996: Formicidae (Insecta: Hymenoptera) of Saudi Arabia (Part 2). Fauna of Saudi Arabia 15: 300-385.

Collingwood, C. A.; Tigar, B. J. \& Agosti, D. 1997: Introduced ants in the United Arab Emirates. Journal of Arid Environments 37: 505-512.

Collingwood, C. A. \& Harten, A. van 2001: Additions to the ant fauna of Yemen. (Hymenoptera, Formicidae). - Esperiana 8: 559-568.

Collingwood, C. A.; Agosti, D.; Sharaf, M. R. \& van Harten, T. 2011: Order Hymenoptera, Family Formicidae. - Arthropod Fauna of the UAE 4: 405-474.

Dezhakam, M. \& Soleyman Nejadiyan, E. 2000: The fauna of symbiotic ants with the southern mealybug, Nipaecoccus virids, on citrus in Khuzestan. Proceedings of 14th Iranian Plant Protection Congress, Isfahan University of Technology: p. 266.

Dlussky, G. M. 1967: Murawoi roda formica du SSR. Moscow: Nauka: 231 pp.

Dlussky, G. M.; Soyunov, O. S. \& Zabelin, S. I. 1990: Ants of Turkmenistan. [in Russian]. - Ashkabad: Ylym Press: 273 pp.

EMERY, C. 1908: Beiträge zur Monographie der Formiciden des paläarktischen Faunengebietes (Hym.) (Fortsetzung.) III. Die mit Aphaenogaster verwandte Gattungengruppe. - Deutsche Entomologische Zeitschrift 1908: 305-338.

EMERY, C. 1915: Escursioni zoologiche del Dr. Enrico Festa nell'Isola di Rodi. XII. Formiche. - Bollettino dei Musei di Zoologia ed Anatomia Comparata della Reale Università di Torino 30 (701): 1-7.

Firouzi, F.; Pashaei Rad, Sh.; Hossein Nezhad, S. \& Agosti, D. 2011: Four new records of ants from Iran (Hymenoptera: Formicidae). - Zoology in the Middle East 52: 71-78.

FOREL, A. 1895: Südpalaearctische Ameisen. - Mitteilungen der Schweizerischen Entomologischen Gesellschaft 9: 227-234.

Forel, A. 1904: Dimorphisme du mâle chez les fourmis et quelques autres notices myrmécologiques. - Annales de la Société Entomologique de Belgique 48: 421-425.

Forel, A. 1907: Formicides du Musée National Hongrois. - Annales Historico-Naturales Musei Nationalis Hungarici 5: 1-42.

Ghahari, H. \& Collingwood, C. A. 2011: A study on the ants (Hymenoptera: Formicidae) of southern Iran. - Calodema 176: 1-5.

Ghahari, H. \& Collingwood, C. A. 2013: A study on the ants (Hymenoptera: Vespoidea: Formicidae) from western Iran. - Acta Phytopathologica et Entomologica Hungarica 48 (1): 155-164.

Ghahari, H.; Ostovan, H.; Kamali, K. \& Tabari, M. 2008: Arthropod predators of rice fields in central parts of Mazandaran. - Journal of Agricultural Sciences (Islamic Azad University) 14 (1): 63-74. 
Ghahari, H.; Collingwood, C. A.; Tabari, M. \& Ostovan, H. 2009: Faunistic notes on Formicidae (Insecta: Hymenoptera) of rice fields and surrounding grasslands of northern Iran. - Munis Entomology \& Zoology 4 (1): 184-189.

Ghahari, H.; Collingwood, C. A.; Sakenin, H. ～\& Ostovan, H. 2010a: A contribution to the ants (Hymenoptera: Formicidae) of some regions in southern Iran. - Proceedings of ISSAAS 2009 (The International Society for Southeast Asian Agricultural Sciences), January 11-15, 2010 Pattaya, Thailand: p. 183.

Ghahari, H.; Tabari, M.; Rashidi, A. \& Mohebbi, H. R. 2010b: Faunistic survey and population fluctuations of ants (Hymenoptera: Formicidae) in rice fields of Mazandaran. - Journal of New Agricultural Science (Modern Science of Sustainable Agriculture) 6 (19): 61-70 (in Persian with English summary).

Ghahari, H.; Collingwood, C. A.; Havaskary, M.; Ostovan, H. \& SAmin, N. 2011: A contribution to the knowledge of ants (Hymenoptera: Formicidae) from the Arasbaran biosphere reserve and vicinity, northwestern Iran. - Jordan Journal of Agricultural Sciences 7 (3): 558-563.

Gholami, M.; Afshari, A. \& Mafi Pashakolaei, SH. A. 2012: The fauna and frequency of cottony cushion scale (Icerya purchasi MAsKeLL) - related ants (Hymenoptera: Formicidae) community in citrus orchards of Sari region, northern Iran. - Proceedings of 20th Iranian Plant Protection Congress, University of Shiraz: p. 175.

Holway, D. A.; Lach, L.; Suarez, A. V.; Tsutsui, N. D. \& CAse, T. J. 2002: The causes and consequences of ant invasions. - Annual Review of Ecology and Systematics 33: 181-233.

Hölldobler, B. \& Wilson, E. O. 1990: The ants. Cambridge, Mass.: Harvard University Press: xii + $732 \mathrm{pp}$.

Hossein Nezhad, S.; Pashaei Rad, Sh.; Firouzi, F. \& Agosti, D. 2012: New and additional records for the ant fauna from Iran (Hymenoptera: Formicidae). Zoology in the Middle East 55: 65-74.

IONESCU-Hirsch, A. 2009: An annotated list of Camponotus of Israel (Hymenoptera: Formicidae), with a key and descriptions of new species. - Israel Journal of Entomology 39: 57-98.

Karaman, C. \& Aktac, N. 2013: Descriptions of four New Species of Camponotus MaYr (Hymenoptera: Formicidae), with a Key for the Worker Caste of the Camponotus of Turkey. - Journal of the Kansas Entomological Society 86 (1): 36-56.

Mayr, G. 1877: Formicidae. [In Russian]. - In: Fedchenko, A. P. 1877: Travels in Turkestan. Vol. 2, Div. 5, No. 7. [In Russian.]. - Izvestiya Imperatorskago Obshchestva Lyubitelei Estestvoznaniya Antropologii i Etnografii pri Imperatorskom Moskovskom Universitete 26: i-iii, $1-20(+1)$.
Menozzi, C. 1927: Zur Erforschung des Persischen Golfes (Beitrag nr. 12) Formicidae (Hym.). - Supplementa Entomologica 16: 117-119.

Mirseyed, S.; Asgari, Sh. \& SAmin, N. 2013: A study on the natural enemies of coccids (Hemiptera: Coccidae) in pistachio gardens of Damghan, Iran. - Calodema 281: $1-5$.

Modarres Awal, M. 1997: Family Formicidae: p. 274. - In: Modarres Awal, M. (ed.): List of agricultural pests and their natural enemies in Iran. - Ferdowsi University Press: 429 pp.

Mohammadi, S.; Mossadegh, M. S. \& Esfandiari, M. 2012: Eight ants species (Hymenoptera: Formicidae) new for the fauna of Iran. - Munis Entomology \& Zoology 7 (2): 847-851.

Mossadegh, M. S. 2012: Symbiosis of symbiotic ants of Nipaecoccus viridis (New.) in citrus orchard of Khuzestan. - Proceedings of 20th Iranian Plant Protection Congress, University of Shiraz: p. 823.

Mossadegh, M. S.; Esfandiari, M. \& Heidarynia, Z. 2008: The relationship effects of symbiotic ants on biological control Nipaecoccus viridis (NEw.) by Cryptolaemous montrouzieri MuL. in citrus orchards of north Khuzestan. - Proceedings of 18th Iranian Plant Protection Congress, University of Bu-Ali Sina Hamadan: p. 63.

PAKnia, O. \& Kami, H. G. 2007: New and additional record for Formicidae (Hymenoptera: Insecta) fauna of Iran. - Zoology in the Middle East 40: 85-90.

Paknia, O. \& Tirgari, S. 2004: Study of fauna and geographical distribution of stinging ants (Hymenoptera: Formicidae) and their medical importance in Lar, Iran. - Proceedings of 16th Iranian Plant Protection Congress, University of Tabriz: p. 293.

PAKniA, O. 2006: Distribution of the introduced ponerine ant, Pachycondyla sennaarensis (Hymenoptera: Formicidae) in Iran. - Myrmecologische Nachrichten 8: 235-238.

Paknia, O.; Radchenko, A.; Alipanah, H. \& PfeifFER, M. 2008: A preliminary checklist of the ants (Hymenoptera: Formicidae) of Iran. - Myrmecological News 11: 151-159.

Paknia, O.; Radchenko, A. G. \& Pfeiffer, M. 2010: New records of ants (Hymenoptera: Formicidae) from Iran. - Asian Myrmecology 3: 29-38.

PISARSKI, B. 1967: Fourmis (Hymenoptera: Formicidae) d'Afghanistan récoltées par M. Dr. K. LiNDBERG. Annales Zoologici (Warsaw) 24: 375-425.

Radchenko, A. G. 1996: A key to the ant genus Camponotus (Hymenoptera, Formicidae) in Palearctic Asia. [in Russian]. - Zoologicheskii Zhurnal 75: 1195-1203.

RADChenko, A. G. 1997: A review of ants of the subgenera Tanaemyrmex, Colobopsis Myrmablis, Myrmosericus, Orthonotomyrmex and Paramyrmablis of the genus Camponotus MAYr (Hymenoptera: Formicidae) of the Asian part of Palearctic. - Zoologicheskii Zhurnal 76: 805-815 (in Russian). 
Radchenko, A. G. 1998: A key to the ants of the genus Cataglyphis Foerster (Hymenoptera, Formicidae) of Asia. [in Russian]. - Entomologicheskoe Obozrenie 77: 502-508.

Radchenko, A. \& Paknia, O. 2010: Two new species of the genus Cataglyphis Foerster, 1850 (Hymenoptera: Formicidae) from Iran. - Annales Zoologici (Warszawa) 60 (1): 69-76.

Rafinejad, J.; Zareit, A.; Akbarzadeh, K.; Azad, M.; Biglaryan, F.; Doosti, S. \& Sedaghat, M. M. 2009: Faunestic study of ants with emphasis on the health risk of stinging ants in Qeshm Island, Iran. - Iranian Journal Arthropod-Borne Diseases 3 (1): 53-59.

Ruzsky, M. 1896: Verzeichniss der Ameisen des östlichen Russlands und des Uralgebirges. - Berliner Entomologische Zeitschrift 41: 67-74.

Sakenin Chelav, H.; Eslami, B.; Samin, N.; Imani, S.; Shirdel, F. \& Havaskary, M. 2008a: A contribution to the most important trees and shrubs as the hosts of wood-boring beetles in different regions of Iran and identification of many natural enemies. - Journal of Plant and Ecosystem 16: 27-46.

Sakenin Chelav, H.; Ghahari, H.; Tabari, M.; Monem, R.; Havaskary, M. \& Rashidi, A. 2008b: A preliminary survey on the fauna of ants (Hymenoptera: Formicidae, Mutillidae) and rove beetles (Coleoptera: Staphylinidae) in Iranian rice fields and surrounding grasslands. - Proceedings of National Conference of Agronomical Rice Breeding, Young Research Club Islamic Azad University of Ghaemshahr, 26-27 November: p. 80.

SAmin, N.; Zhou, H.; Imani, S. \& Rastegar, J. 2011: A contribution to the knowledge of Iranian Staphylinidae (Coleoptera: Staphylinoidea). - Archive Biological Sciences, Belgrade 63 (4): 1235-1243.

SANTSCHI, F. 1911: Formicides de diverses provenances. - Annales de la Societe Entomologique de Belgique 55: 278-287.

SANTSCHI, F. 1921: Notes sur les fourmis paléarctiques. II. Fourmis d'Asie Mineure récoltées par M. H. Gadeau de Kerville. - Boletín de la Real Sociedad Española de Historia Natural 21: 110-116.

SANTSCHI, F. 1927a: Révision myrmécologique. - Bulletin et Annales de la Société Entomologique de Belgique 67: 240-248.

SANTSCHI, F. 1929: Étude sur les Cataglyphis. - Revue Suisse de Zoologie 36: 25-70.

Seifert, B. \& Schultz, R. 2009: A taxonomic revision of the Formica rufibarbis FABRICIUS, 1793 group (Hymenoptera: Formicidae). - Myrmecologische Nachrichten 12: 255-272.

Sharaf, M. R. 2006: Taxonomic and ecological studies on family Formicidae (Order: Hymenoptera) in Egypt including some protectorates with a study of some insect fauna associated with ant species. - Ain Shams University, Faculty of Science, Entomology Department, Cairo (unpublished thesis): 340 pp.
Shattuck, S. O. 1994: Taxonomic catalog of the ant subfamilies Aneuretinae and Dolichoderinae (Hymenoptera: Formicidae). - University of California Publications in Entomology 112: i-xix, 1-241.

Shiran, E.; Mossadegh, M. S. \& Esfandiari, M. 2012: Symbiotic ants with aphids in Khuzestan Province, Isfahan city and its vicinity, Iran. - Proceedings of 20th Iranian Plant Protection Congress, University of Shiraz: p. 183.

Shiran, E.; Mossadegh, M. S. \& Esfandiari, M. 2013: Mutualistic ants (Hymenoptera: Formicidae) associated with aphids in central and southwestern parts of Iran. - Journal of Crop Protection 2 (1): 1-12.

Sмith, F. 1858: Catalogue of hymenopterous insects in the collection of the British Museum. Part VI. Formicidae. - London: British Museum: 216 pp.

Suarez, A. V.; Bolger, D. T. \& CASE, T. J. 1998: Effects of fragmentation and invasion on native ant communities in coastal southern California. - Ecology $79(6)$ : 2041-2056.

Tirgari, S. \& Paknia, O. 2004: Additional records for the Iranian fauna of Formicidae (Hymenoptera). Zoology in the Middle East 32: 115-116.

Tонмé, G. \& Toнmé, H. 1981: Les fourmis du genre Messor en Syrie. Position systématique. Description de quelques ailés et de formes nouvelles. Répartition géographique. - Ecologia Mediterranea 7 (1): 139-153.

WARD, P. S. 1987: Distribution of the introduced Argentine ant (Iridomyrmex humilis) in natural habitats of the Lower Sacramento Valley and its effects on the indigenous ant fauna. - Hilgardia 55 (2): 1-16.

WheEler, W. M. 1930-1931: List of the known Chinese ants. - Peking Natural History Bulletin 5: 53-81.

Wilson, E. O. 2000: Foreword. - In: Agosti, D.; Majer, J. D.; Alonso, L. E. \& Schultz, T. R. (eds.) 2000: Ants. Standard methods for measuring and monitoring biodiversity. - Washington: Smithsonian Institution Press: xix +280 pp.

Zehzad, B.; KiABI, B. H. \& Madjnoonian, H. 2002: The natural areas and landscape of Iran: an overview. Zoology in the Middle East 26: 7-10. 

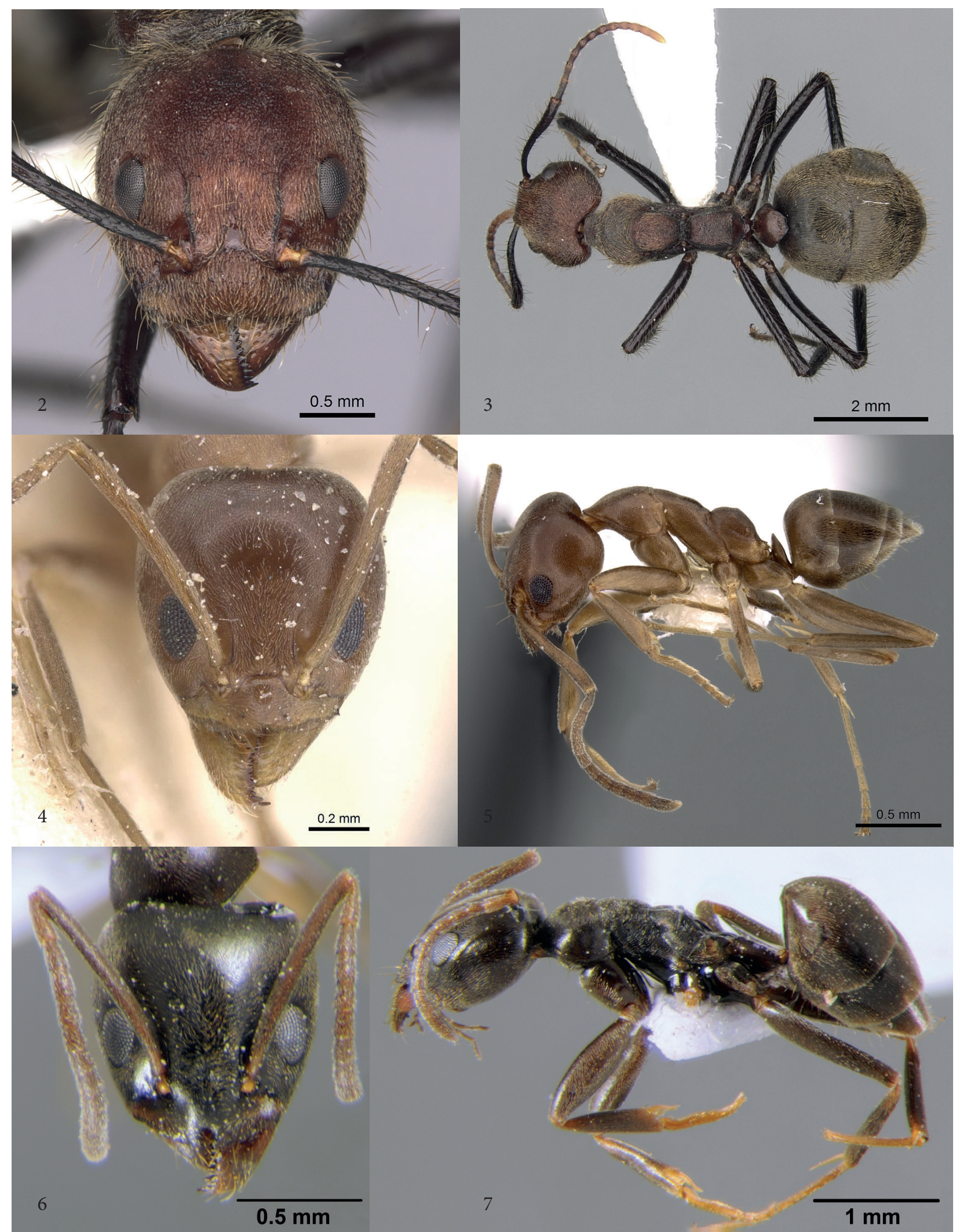

Figs 2-7: 2, 3 Dolichoderus quadripunctatus (casent0246569, Italy), 2 Head in full-face view, 3 Body in profile, Photographer: Andrea Walker; 4, 5 Linepithema humile (casent0106119, Argentina), 4 Head in full-face view, 5 Body in profile, Photographer: Michael Branstetter: 6, 7 Tapinoma simrothi (casent0910228, Argentina), 6 Head in full-face view, 7 Body in profile, Photographer: Michael Branstetter. 


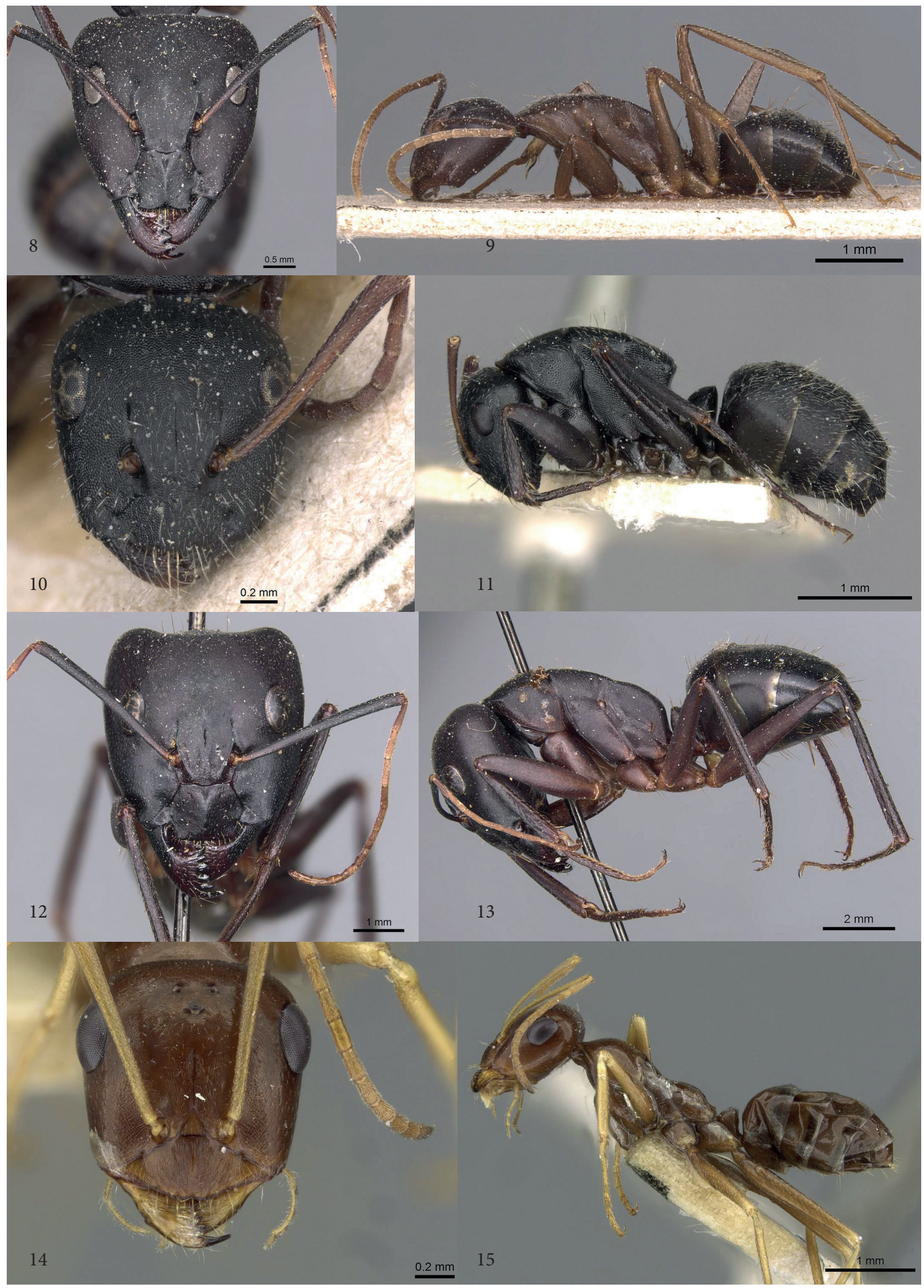




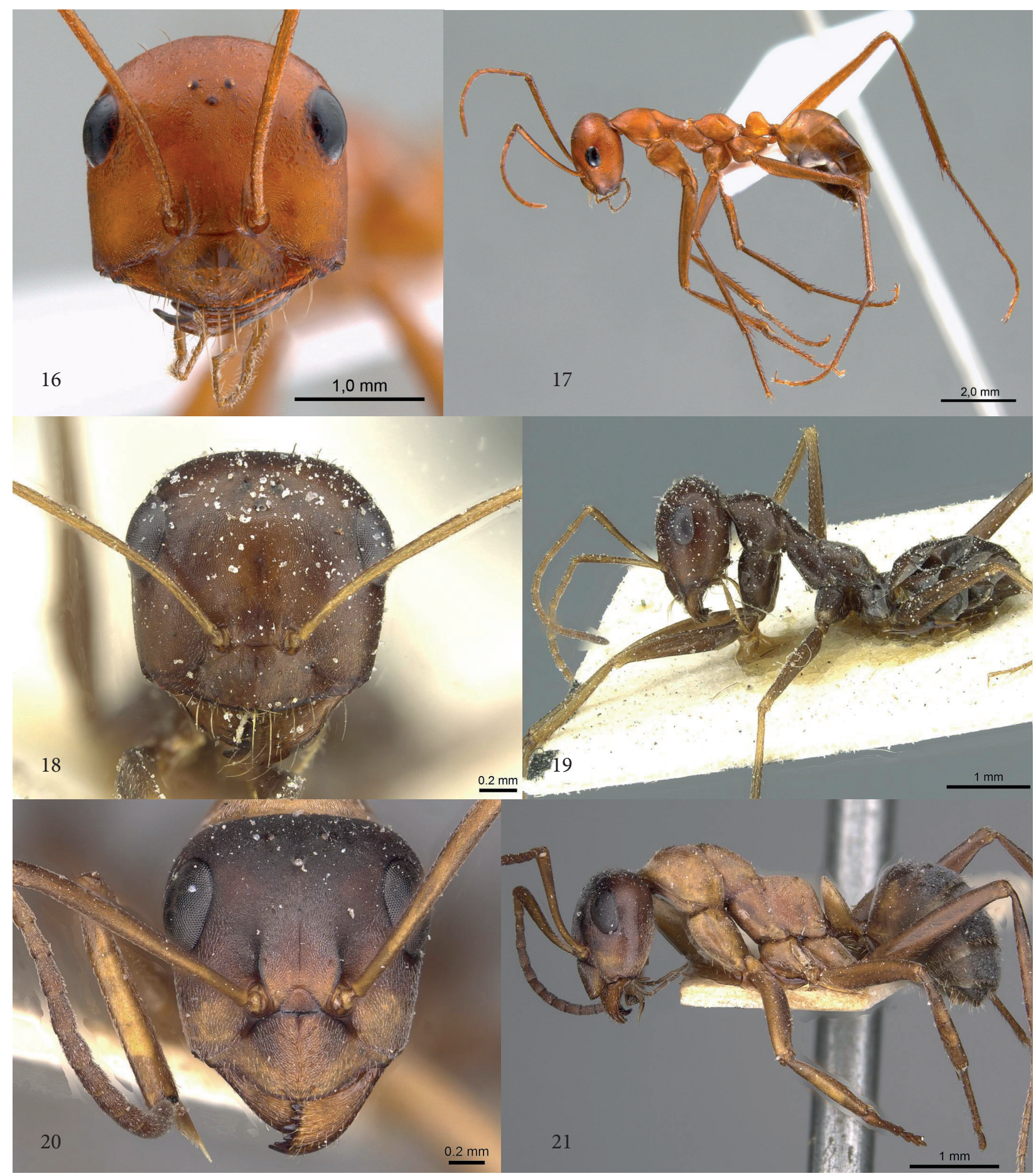

Figs 16-21: 16, 17 Cataglyphis nodus (casent0906069, Holotype); 16 Head in full-face view, 17 Body in profile, Photographer: Shannon Hartman; 18, 19 Cataglyphis semitonsa (casent0912230, Holotype), 18 Head in full-face view, 19 Body in profile, Photographer: Zach Lieberman; 20, 21 Formica glauca (casent0911078, Holotype), 20 Head in full-face view, 21 Body in profile, Photographer: Will Ericson.

Figs 8-15 (oposite): 8, 9 Camponotus adenensis (casent0905283, Holotype); 8 Head in full-face view, 9 Body in profile, Photographer: Will Ericson; 10, 11 Camponotus libanicus (casent0913700, Holotype), 10 Head in full-face view, 11 Body in profile, Photographer: Alexandra Westrich; 12, 13 Camponotus xerxes (casent0905292, Holotype), 12 Head in full-face view, 13 Body in profile, Photographer: Will Ericson; 14, 15 Cataglyphis cinnamomea (casent0911105, Holotype), 14 Head in full-face view, 15 Body in profile, Photographer: Zach Lieberman. 


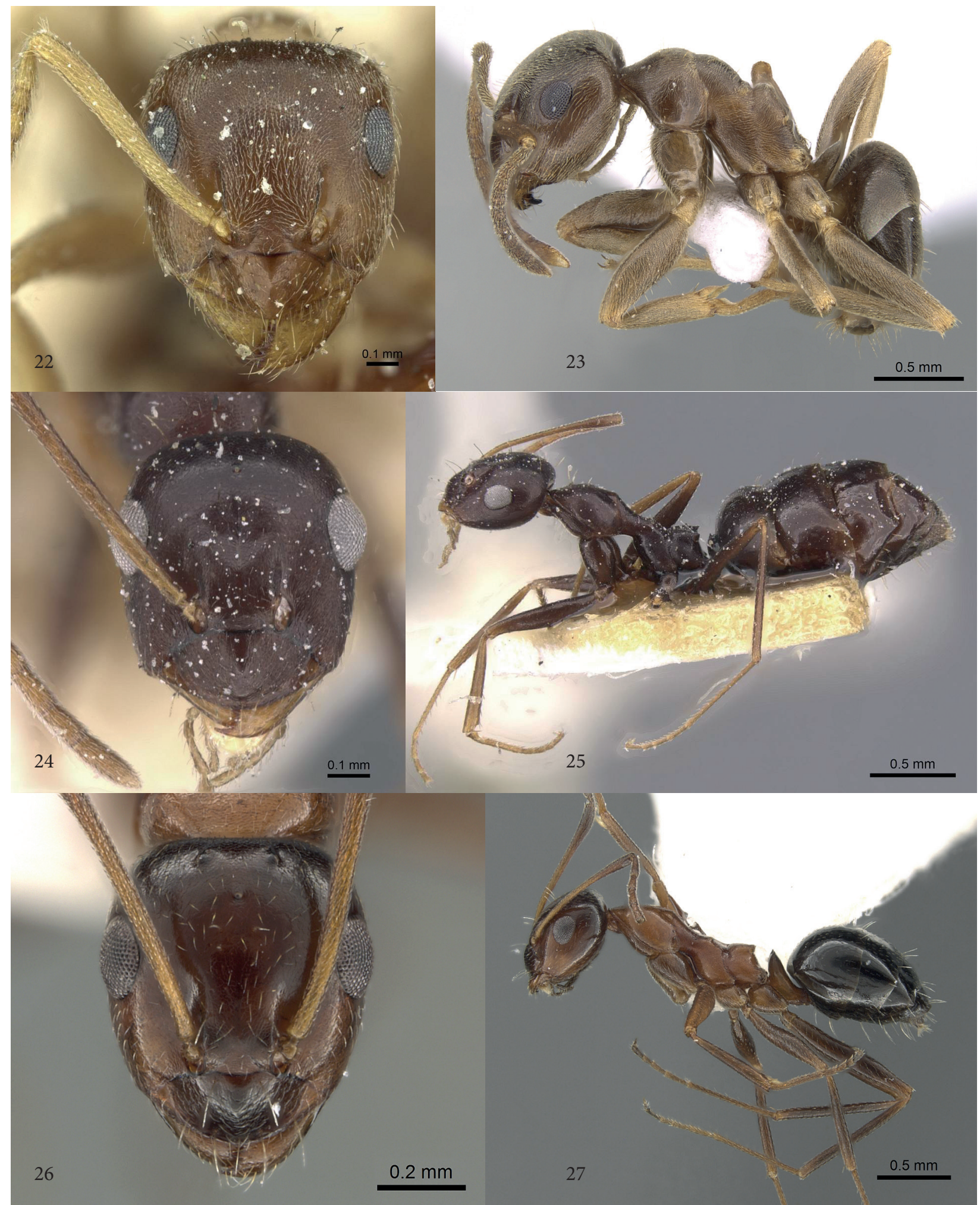

Figs 22-27: 22, 23 Lasius turcicus (casent0912297, Holotype), 22 Head in full-face view, 23 Body in profile, Photographer: Zach Lieberman; 24, 25 Lepisiota karawaiewi (casent0912405, Holotype); 24 Head in full-face view, 25 Body in profile, Photographer: Will Ericson; 26,27 Lepisiota melas (casent0905146, Holotype), 26 Head in full-face view, 27 Body in profile, Photographer: Zach Lieberman. 


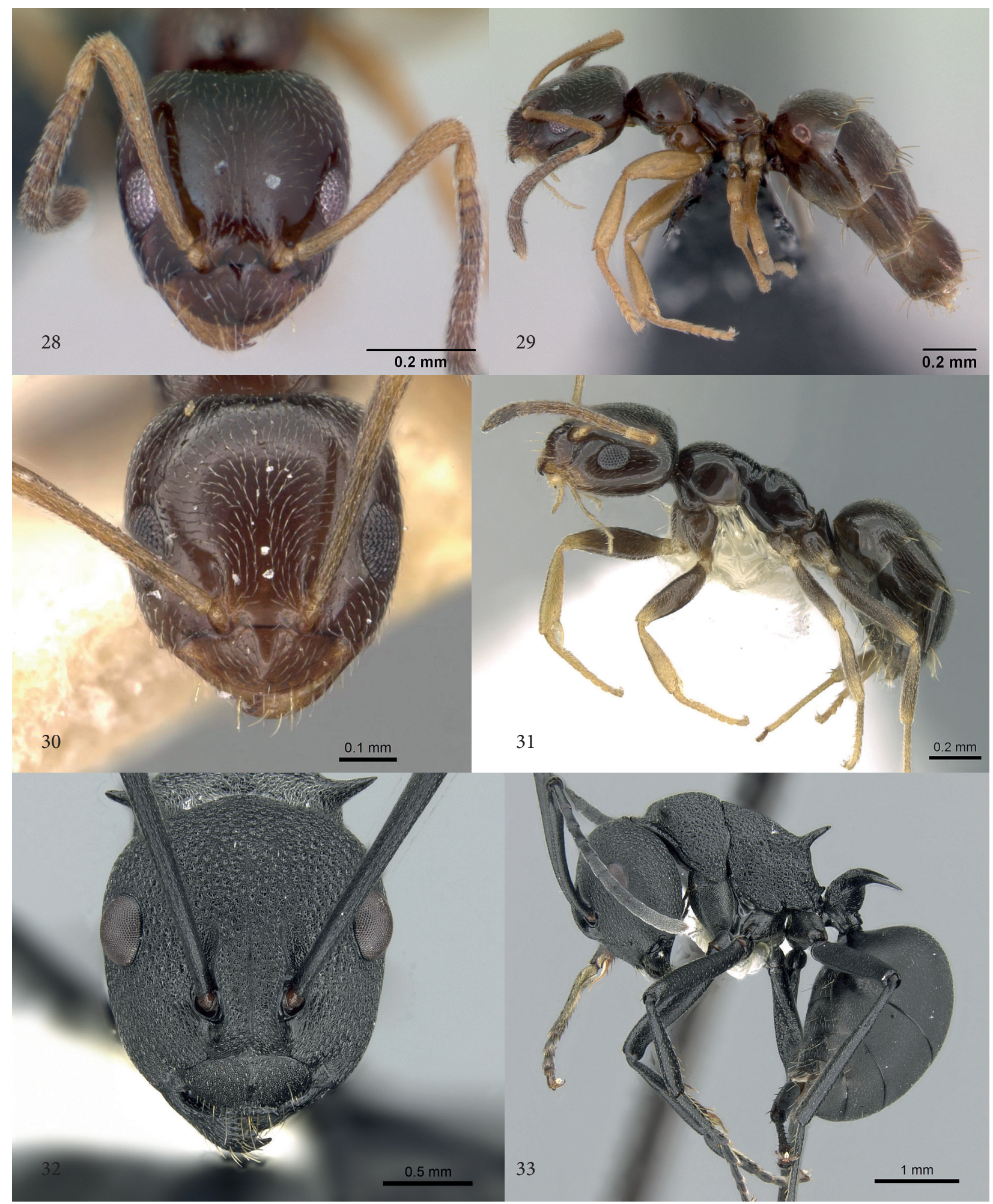

Figs 28-33: 28, 29 Plagiolepis pygmaea (casent0912420, Holotype), 28 Head in full-face view, 29 Body in profile, Photographer: Zach Lieberman; 30, 31 Plagiolepis schmitzii (casent0909859, Holotype), 30 Head in full-face view, 31 Body in profile, Photographer: Will Ericson; 32, 33 Polyrhachis lacteipennis (casent0281400, Nepal); 32 Head in full-face view, 33 Body in profile, Photographer: Michele Esposito. 


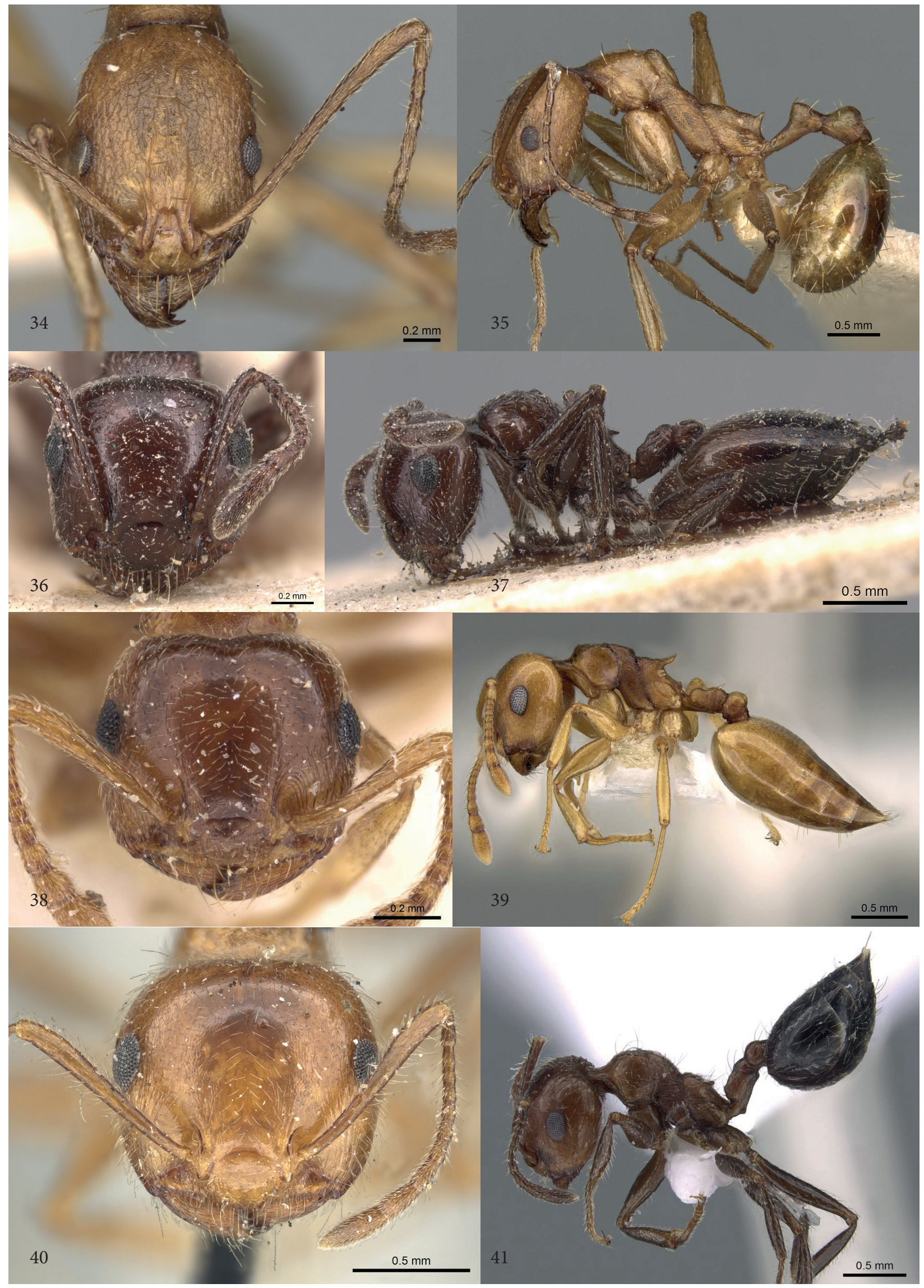




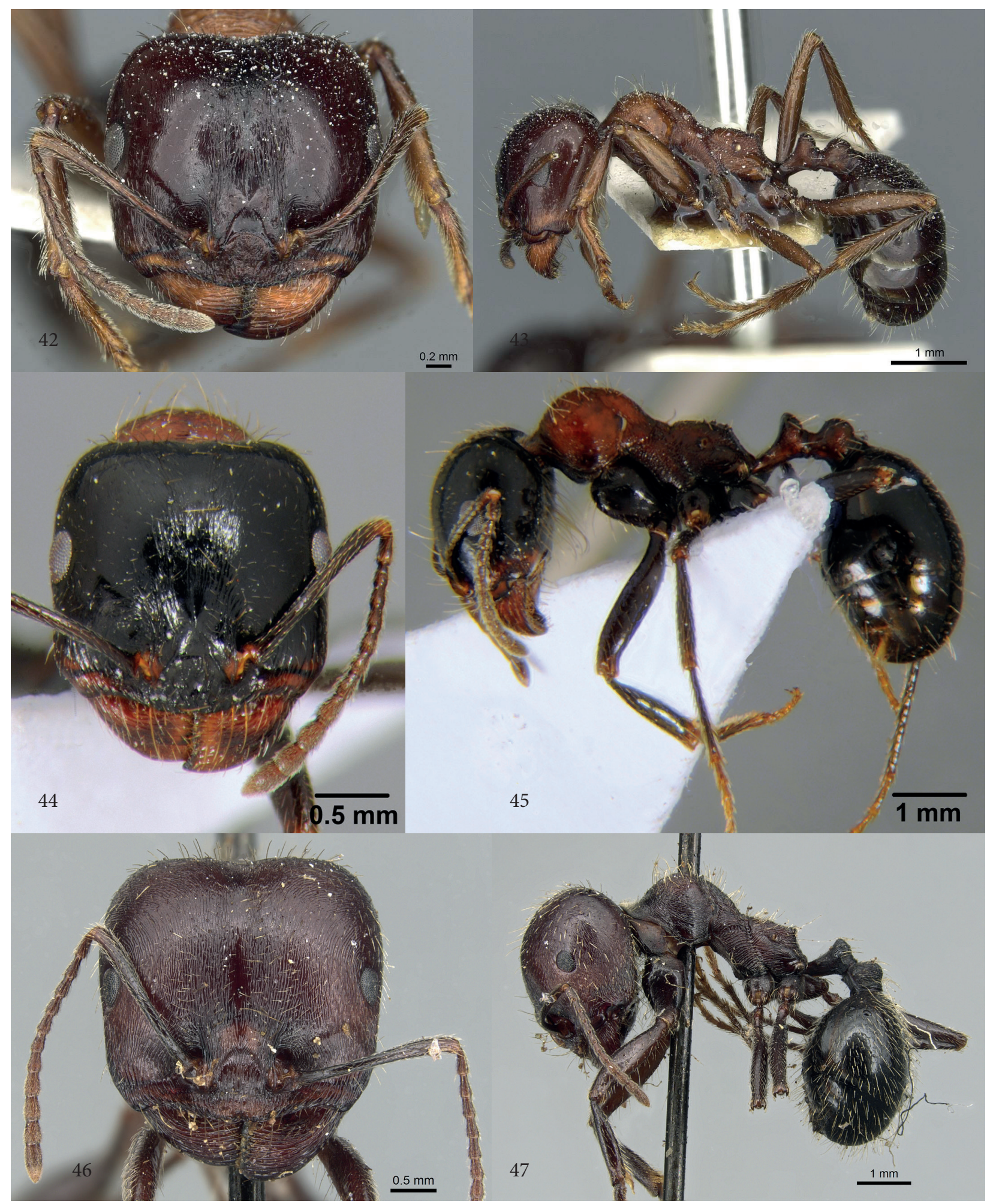

Figs 42-47: 42, 43 Messor denticulatus (casent0913176, Holotype), 42 Head in full-face view, 43 Body in profile, Photographer: Zach Lieberman; 44, 45 Messor meridionalis (CFH000073, Iran), 44 Head in full-face view, 45 Body in profile, Photographer: Donat Agosti; 46, 47 Messor orientalis (casent0904132, Holotype), 46 Head in full-face view, 47 Body in profile, Photographer: Will Ericson.

Figs 34-41(oposite): 34, 35 Aphaenogaster splendida (casent0280965, Holotype), 34 Head in full-face view, 35 Body in profile, Photographer: Shannon Hartman; 36, 37 Crematogaster laestrygon (casent0904500, Holotype), 36 Head in full-face view, 37 Body in profile, Photographer: Will Ericson; 38, 39 Crematogaster luctans (casent0908611, Holotype), 38 Head in full-face view, 39 Body in profile, Photographer: Zach Lieberman; 40, 41 Crematogaster subdentata (casent0902140, Holotype); 40 Head in full-face view, 41 Body in profile, Photographer: Ryan Perry. 


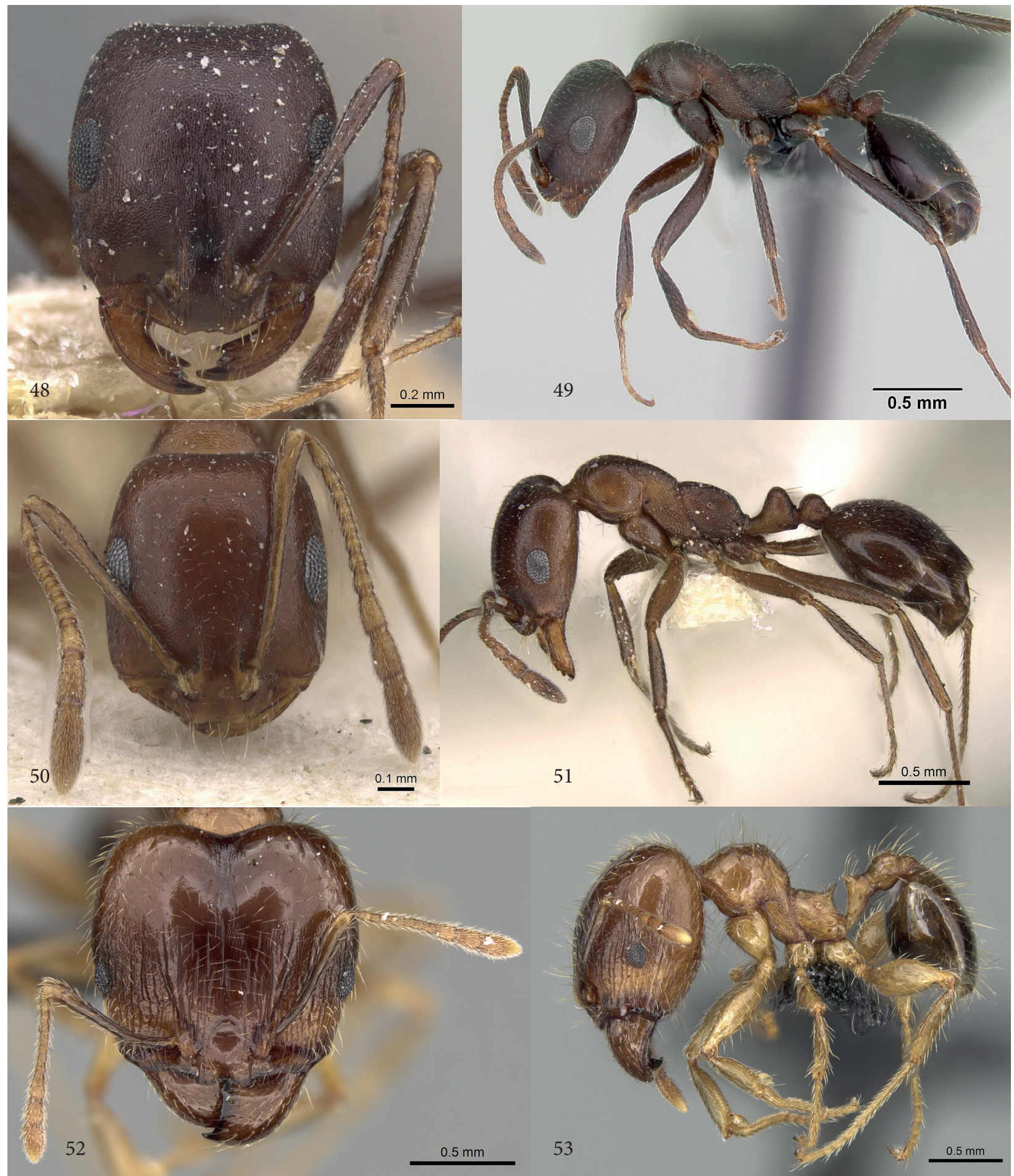

Figs 48-53: 48, 49 Monomorium areniphilum (casent0913555, Holotype); 48 Head in full-face view, 49 Body in profile, Photographer: Will Ericson; 50, 51 Monomorium salomonis (casent0913835, Holotype), 50 Head in full-face view, 51 Body in profile, Photographer: Will Ericson; 52, 53 Mesoponera ambigua (casent0006209, Madagascar), 52 Head in full-face view, 53 Body in profile, Photographer: April Nobile. 


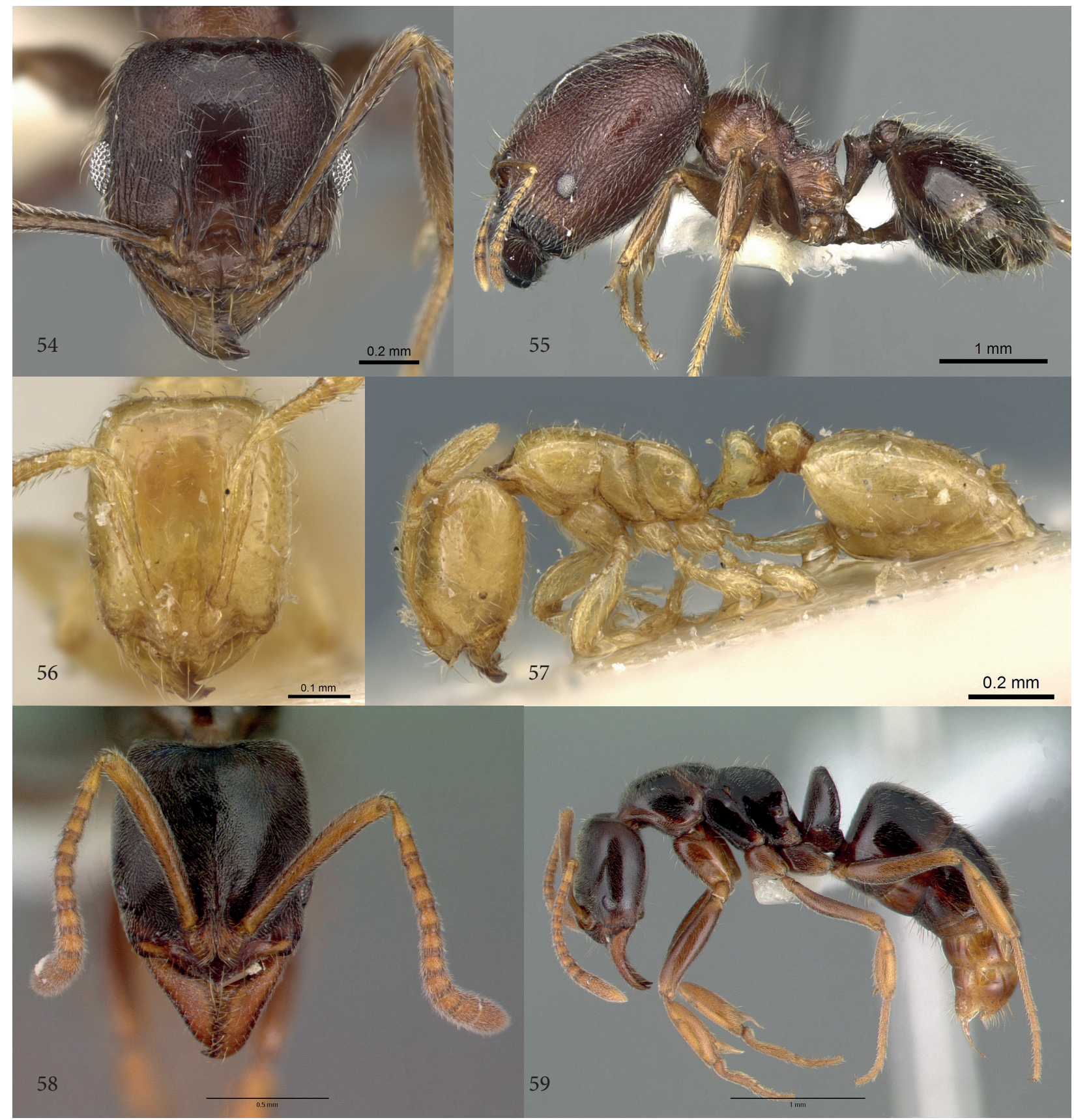

Figs 54-59: 54, 55 Pheidole megacephala (casent0104990, Neotype, Mauritius), 54 Head in full-face view, 55 Body in profile, Photographer: Michele Esposito; 56, 57 Pheidole sculpturata (casent0281616, Botswana); 56 Head in full-face view, 57 Body in profile, Photographer: Michele Esposito; 58, 59: Solenopsis wolfi (casent0904622, Holotype), 58 Head in full-face view, 59 Body in profile, Photographer: Zach Lieberman. 